\title{
Abstracts
}

\section{Physical Activity/Participation Session 1}

\author{
Physical Behavior is Associated with Age and \\ Ambulatory Status, But Not with Gender or \\ VO $_{2 p e a k}$ in Wheelchair-using Youth with Spina \\ Bifida \\ Bloemen, Manon ${ }^{1,2}$, Takken, Tim ${ }^{2}$, de Groot, Janke ${ }^{1,2}$, \\ Kruitwagen, $\mathrm{Cas}^{2}$, Backx, Frank ${ }^{2}$ \\ ${ }^{1}$ HU University of Applied Sciences Utrecht \\ ${ }^{2}$ University Medical Center Utrecht
}

Background Physical behavior (PB: expressed as type of activities and intensity) of wheelchair-using youth with Spina Bifida (SB) is unfavorable compared to peers. Evidence shows relationships between $\mathrm{PB}$ and age, gender and $\mathrm{VO}_{2 \text { peak }}$ in typically developing youth and PB and ambulatory status in youth with Cerebral Palsy. The aim of this study was to analyze the associations between $\mathrm{PB}$ and age, gender, $\mathrm{VO}_{2 \text { peak }}$ and ambulatory status in wheelchair-using youth with SB.

Methods VitaMove data of 34 and Actiheart data of 36 wheelchair-using (for daily life, sports or long distances) youth with SB were used to assess PB. The VitaMove measures the type of activities. Sedentary activities were defined as sitting and lying and dynamic activities as walking, running, wheeling, (hand)biking and non-cyclic moving. The Actiheart measures the intensity. Time spent in Moderate to Vigorous Physical Activity (MVPA) was analyzed. The Shuttle Ride Test measured $\mathrm{VO}_{2 \text { peak }}$ and the Hoffer classification measured ambulatory status. Univariate and multivariate regression analyses were performed with PB (sedentary activities, dynamic activities, MVPA) as the dependent variable. Independent variables were age, gender, $\mathrm{VO}_{2 \text { peak }}$ and Hoffer (Hoffer 1-3 vs 4-5).

Results Sedentary and dynamic activities during a school day are influenced by both age and Hoffer. During a weekend day, sedentary and dynamic activities are influenced by Hoffer alone. MVPA is influenced by Hoffer during a school day and by age during a weekend day. Overall, participants with Hoffer 4-5 are per- forming worse than Hoffer 1-3 and older participants performing worse than younger participants.

Conclusions PB is associated with age and ambulatory status in wheelchair-using youth with SB, with older age and the inability to walk influencing PB negatively. Gender and $\mathrm{VO}_{2 \text { peak }}$ are not associated with $\mathrm{PB}$ in wheelchair-using youth with SB.

\section{Can Initial Assessment Findings Predict Future Mobility of Children with Spina Bifida? \\ Botman, Antoinette $^{1}$, Taylor, Suzie ${ }^{1}$, Quinlan, Rachal $^{1}$, Harris, Ryan ${ }^{2}$, Paulka, Michael ${ }^{2}$, Pacey, Verity $^{2,1}$ \\ ${ }^{1}$ The Sydney Children's Hospitals Network \\ ${ }^{2}$ Macquarie University}

Background Spina Bifida (SB) is a neural tube defect that affects 4.6/10,000 Australian births/year. A major barrier to participation and quality of life in individuals with SB is their decreased mobility. Current evidence suggests motor lesion level, orthopaedic surgery and ventriculoperitoneal shunt (VPS) history predict future mobility in children with SB. This study aims to determine whether physical assessment findings and comorbidities recorded before three months of age can predict the peak mobility level a child with SB can achieve prior to starting school.

Methods Retrospective data analysis was undertaken on all children with SB managed at the Children's Hospital at Westmead between 2005-2015.54 children (34 male) with lumbosacral level SB (37 myelomeningocoele, 14 lipomeningocoele, 3 meningocoele) were included. Medical records were audited and data collected on modified manual muscle testing results, congenital lower limb deformity, demographic and medical characteristics. Stepwise multiple regression was undertaken to ascertain whether any combination of findings could predict future peak mobility. 
Results Hip and knee extensor strength at initial assessment predicted $51 \%$ of the variance in the peak mobility a child achieved before starting school, assessed with the Hoffer Scale. The addition of VPS status raised the predictability to $62 \%$ of the variance when the Hoffer Scale was modified to account for orthotic and walking aide use (all $p<0.05$ ).

Conclusions The presence of a VPS and hip, knee and ankle strength before three months of age can explain significant variance in the future mobility status of a child with SB. These findings may increase clinicians' confidence to predict a neonate's future mobility and assist families and multidisciplinary treatment teams to set realistic mobility goals and assist the child to optimise their mobility potential.

\section{Using Initial Physical Assessment Findings, it is Possible to Predict the Age a Child with Spina Bifida will Commence Independent Walking: A Retrospective Chart Audit}

Botman, Antoinette ${ }^{1}$, Quinlan, Rachal ${ }^{1}$, Taylor, Suzie $^{1}$, Paulka, Michael ${ }^{2}$, Harris, Ryan ${ }^{2}$, Pacey, Verity $^{2,1}$

${ }^{1}$ The Sydney Children's Hospitals Network

${ }^{2}$ Macquarie University

Background The majority of research into predictors of walking in children with Spina Bifida (SB) has focused on the maximum ambulation that is achieved based on physical assessment findings obtained after walking has already commenced. Predictors of age of walking are unknown, but important to inform clinical practice. This study aims to investigate whether physical assessment findings in the first two months of life of a child with SB can predict the age of independent walking. Being able to predict the age that walking can be achieved gives the parents of a newborn child with SB a tangible idea of how their child may function and what to plan for in the future.

Methods Retrospective data of initial modified manual muscle testing results, congenital lower limb abnormalities, and demographic and medical characteristics for 51 children (28 male) with SB managed in the Spina Bifida Service at The Children's Hospital at Westmead, Sydney, Australia (2005-2015) was collected. A stepwise linear regression was performed with the age that walking was achieved as the dependent variable and type of SB, level of SB, congenital abnormality of hip, knee or foot, hydrocephalus, VP shunt, and the presence of neurogenic bladder or bowel as the independent variables.
Results All children in the study achieved independent walking between 9 and 36 months. $83 \%$ of the variance in age of achieving walking was predicted by three factors: ankle plantarflexion strength, VP shunt history and knee extension strength $(p<0.05)$.

Conclusions Children who have better ankle plantarflexion and knee extension strength, in the absence of a VP shunt, will achieve walking earlier in life. A clinical implication identified from this study is the ability of the clinician to better inform parents of children with SB what to expect from a developmental perspective.

\section{Walking Activity in Children with Myelomeningo- cele \\ Yasmeh, Pauline ${ }^{1}$, Mueske, Nicole ${ }^{1}$, Yasmeh, Siamak $^{2}$, Ryan, Deirdre ${ }^{1,2}$, Wren, Tishya ${ }^{1,2}$ \\ ${ }^{1}$ Children's Hospital Los Angeles \\ ${ }^{2}$ University of Southern California}

Background Children with myelomeningocele (MM) may have some degree of spinal cord damage, paralysis, muscle weakness and loss of sensation in the lower extremities ultimately constraining mobility. This study quantified walking activity of children with MM in everyday life.

Methods Walking activity was recorded using a StepWatch activity monitor over 1 week in 47 children with MM (27 males; $9.9 \pm 2.6$ years; 18 sacral, 9 low lumbar, 20 mid-high lumbar) and 7 children with typical development ( 5 males; $11.1 \pm 1.9$ years). Average total steps per day, number of steps and minutes spent at low, medium and high intensity stepping were evaluated. Groups were compared using t-tests and chisquared tests with Bonferroni post-hoc adjustment.

Results Children with sacral and low lumbar MM exhibited no observable differences in age, height, weight or BMI compared to typically developing children. Children with sacral and low lumbar MM took a similar number of steps per day compared to typically developing children (low lumbar: $9658 \pm 5331$, sacral: $9166 \pm 3305$, control: $9589 \pm 3322$ ) and did not differ on steps at any intensity level. Children with mid-high lumbar MM were similar in age, height and weight to typically developing children but exhibited higher BMI percentiles $(84.1 \pm 20.0 ; p=0.04)$ and took fewer total steps per day than all other groups $(4867 \pm 3316$; $p \leqslant 0.04)$. Children with mid-high lumbar MM also spent significantly less time taking steps at all intensities, particularly medium-intensity, than the sacral and low lumbar groups $(p \leqslant 0.07)$. 
Conclusions Children with sacral and low lumbar MM had walking activity similar to typically developing children despite a common need for braces and assistive devices. Children with mid-high lumbar MM were less active, which may lead to heightened risk for secondary health conditions in addition to those associated with MM. Understanding walking activity in youth with MM may aid in developing focused interventions.

\author{
Adaptive Design: Cardboard Equipment Solutions \\ for Toddlers with Spina Bifida \\ Dosa, Nienke $^{1}$, Davis, Timothy ${ }^{2}$, Carr, Donald ${ }^{3}$, \\ Smtih, Teri ${ }^{4}$, McGough, Connor ${ }^{6}$, Evensky, Jesse ${ }^{1}$, \\ Sefick, Peyton ${ }^{1}$, Neville, Lisa ${ }^{5}$, Weber, Monica ${ }^{5}$, \\ Fathers, James ${ }^{3}$ \\ ${ }^{1}$ SUNY Upstate Medical University \\ ${ }^{2}$ SUNY Cortland \\ ${ }^{3}$ Syracuse University \\ ${ }^{4}$ Liberty-Post \\ ${ }^{5}$ Jowonio Preschool \\ ${ }^{6}$ Arise Independent Living \\ Center
}

Background Assistive technology that promotes handeye coordination and autonomous movement has been correlated with improved social and cognitive outcomes in children with mobility impairments, and may impact executive functions in later years. Unfortunately insurers do not consider manufactured products that meet these needs as medically necessary. Manufactured equipment also rarely meets the specific positioning needs of a rapidly growing child. "Adaptive Design" uses low cost materials, such as cardboard, to make custom equipment to facilitate early motor learning, recreation, and social participation.

Methods A group of parents (4), therapists (4), teachers (3), and designers (2) joined forces with clinicians from our regional Spina Bifida center for a series of weekend workshops to create custom solutions for two toddlers with Spina Bifida. Parents identified a design "wish list" during a home visit that was arranged in order to obtain measurements and document need/context for design solutions. After a brief introduction to materials (X-acto knife, paper, glue, tripleply cardboard) and principles of construction, parentclinician-designer-maker teams gathered to brainstorm and make design solutions. This was an iterative process. All materials were donated and all participants volunteered their time.
Results Fifteen designs were created over the course of the three workshops. Examples include a para-podium to encourage weight shifting; a custom tilt chair with fold-out soccer table to promote hand-eye coordination; and a seating insert for an up-cycled power wheelchair that provided early access to autonomous mobility.

Conclusions Rapid prototyping using a hands-on approach and co-creation is a feasible and affordable alternative to manufactured equipment for toddlers with Spina Bifida. The "Adaptive Design" paradigm empowers families in the design and making process, strengthens social networks, and builds community.

\section{Nontraditional Medicine \\ Hopson, Betsy D., Graham, Anna, Arynchyna, \\ Anastasia \\ UAB-Children's of Alabama}

Background For the last five years (2012-2016), SBA of AL and Children's of Alabama partnered to host a camp aimed at creating an alternative form of medicine for patients with Spina Bifida. Each summer, patients and their families come together for a weekend camp to experience life changing activities and build long lasting friendships. The main purpose is to give a sense of independence through fun and educational sessions.

Methods Some of the camp activities include adaptive water skiing, water tubing, painting, putt-putt golf, fishing, and pool/dance socials. Educational sessions include wound care, exercise, and self-catheterization teaching. After 4 years of having a pediatric camp, an adult camp was established. The adult camp consists of the same activities as well as a cooking class, selfcare, motivation/social interaction and career building sessions. Each session in led by an adult with SB.

Results The excitement that campers have when they conquer obstacles is truly life-changing not only for the campers but also for the volunteers. After watching their peers do an activity, they gain confidence to do things they never thought possible. They credit camp for having the confidence to obtain a driver's license, start college, and begin managing their finances. We have collected feedback through social media on how camp has enhanced their life. Since camp the young adults have formed a social group that meets monthly. There is now a peer-to-peer support system where the adults are providing support to the children and where parents are connected. To date 155 families have experienced camp. 
Conclusions The camp model has shown the motivation individuals with SB gain from each other has a greater impact than hearing it in the clinic setting. The camp setting provides a non-threatening atmosphere to learn and gain new confidence and skills. We are searching for ways to bring the camp experience into the clinic through peer support and group visits.

\section{Determinants of Physical Activity Participation in Adults with Spina Bifida \\ Vanderbom, Kerri A. \\ University of Alabama at Birmingham}

Background The health benefits of physical activity (PA) are well documented and PA is a leading indicator of health associated with decreased mortality and morbidity. However, a sedentary lifestyle is common for individuals with Spina Bifida (SB), thus placing individuals at greater risk of experiencing secondary and chronic conditions. The purpose of this study was to examine factors that predict PA participation for adults with SB.

Methods An online, national survey was conducted with participants recruited from the National Spina Bifida Association, SB clinics, and social media. Survey measures included: demographics, 5 eligibility questions, amount of weekly PA, PA barriers, self-efficacy, and stage of change. A logistical regression was computed to determine which factors predict PA participation.

Results Two hundred sixty one participants with SB were included. $61.7 \%(n=161)$ were not regularly active (stage of change 1-3). After controlling for level of assistance for activities of daily living, sex, and age, persons who were regularly active (stages 4-5) had significantly higher odds of self-efficacy $\left(\chi^{2}(5,209)=\right.$ 57.44, $p<0.001$; AOR: $1.4 ; 95 \%$ CI 1.2, 1.6) and were $33 \%$ less likely to report barriers compared to persons who were not regularly active (AOR: $0.67 ; 95 \%$ CI $0.57,0.78$ ). Females were $61 \%$ less likely to report participating in regular activity when compared to males (AOR: 0.39; 95\% CI 0.20, 0.78).

Conclusions Future interventions should focus on removing barriers to PA and increasing self-efficacy in order to increase the PA behaviors of adults with SB. Furthermore, strategies to increase PA may need to be developed specific for females with SB. 